\title{
Efficient calculation of g-factors for CG-SENSE in high dimensions: noise amplification in random undersampling
}

\author{
Mehmet Akcakaya1*, Tamer A Basha', Warren J Manning ${ }^{1,2}$, Reza Nezafat ${ }^{1}$ \\ From 17th Annual SCMR Scientific Sessions \\ New Orleans, LA, USA. 16-19 January 2014
}

\section{Background}

SENSE [1,2] is one of the most used parallel imaging techniques. In [1], uniform undersampling was employed to efficiently reconstruct an unalised image, whereas in [2], a conjugate gradient-based method (CG-SENSE) was used for reconstruction with arbitrary trajectories. SENSE framework allows the calculation of g-factors, characterizing the noise amplification for a given k-space trajectory and coil configuration [1]. However, calculation of g-factors for arbitrary trajectories in high dimensions is time-consuming [3]. Furthermore, noise characteristics of random undersampling, used in compressed sensing, is not wellunderstood. In this work, we use a Monte-Carlo (MC) method for fast calculation of g-factors for CG-SENSE similar to $[4,5]$ and apply it to random Cartesian undersampling trajectories. Theory: SENSE involves a prewhitening step $[1,2]$, thus without loss of generality, we assume white noise. SENSE reconstruction solves $\min _{\mathrm{m}}\|\mathbf{y}-\mathbf{E m}\| \|_{2}$, where $\mathbf{E}$ is the system matrix, and $\mathbf{y}$ are the undersampled measurements. The $\mathrm{g}$-factor for the $\mathrm{k}^{\text {th }}$ voxel is given by $g_{k}=\sqrt{ }\left(\left[\mathbf{E}^{*} \mathbf{E}\right]^{-1}{ }_{k, k}\left[\mathbf{E}^{* *} \mathbf{E}\right]_{k, k}\right)$. Inverting $\mathbf{E}^{*} \mathbf{E}$ is not feasible in high dimensions. Instead we note the $g_{k}$ corresponds to the $\mathrm{k}^{\text {th }}$ diagonal of the reconstruction noise covariance matrix (for normalized coil sensitivities), where $\mathbf{n}_{\text {recon }}=\left(\mathbf{E}^{*} \mathbf{E}\right)^{-1} \mathbf{E}^{*} \mathbf{n}_{\text {meas }}$, and $\mathbf{n}_{\text {meas }}$ is measurement noise with identity covariance matrix. We calculate the sample correlation matrix using a $\mathrm{MC}$ approach (since sample mean goes to 0$)$, as $1 /(\mathrm{p}-1) \sum_{\mathrm{p}} \mathbf{n}^{\mathrm{p}}$ recon $\left(\mathbf{n}^{\mathrm{p}} \text { recon }\right)^{*}$ for $\mathrm{p}$ instances of $\mathbf{n}_{\text {recon }}$. Note we only calculate and store the diagonal elements of this matrix, significantly increasing efficiency.

'Medicine, Beth Israel Deaconess Medical Center, Harvard Medical School, Boston, Massachusetts, USA

Full list of author information is available at the end of the article

\begin{abstract}
Methods
The MC method was first verified in a numerical simulation, where the g-factor was explicitly calculated for a 2D coil configuration, to determine how many MC simulations suffice. Whole-heart imaging was performed with an isotropic resolution of $1.3 \mathrm{~mm}$ using a 32-channel coil array. Two 4-fold accelerated acquisitions were performed, one with uniform undersampling $(2 \times 2$ in the $\mathrm{k}_{\mathrm{y}}-\mathrm{k}_{\mathrm{z}}$ plane) and one with random undersampling. Coil sensitivity maps were exported. Images were reconstructed using SENSE (for uniform) and CG-SENSE (for both). g-factors were also calculated with the proposed approach.
\end{abstract}

\section{Results}

Figure 1 shows the results of numerical simulations, indicating the method converges in $\sim 50 \mathrm{MC}$ simulations. Figure 2 shows the reconstructions associated with the two undersampling patterns and reconstructions, and the corresponding g-factors respectively. The results exhibit the semi-convergence property for random undersampling but not for uniform. Furthermore, the $\mathrm{g}$-factor for random undersampling is smaller at its convergent point than for uniform.

\section{Conclusions}

g-factors for random undersampling is better than those for uniform at high $\mathrm{k}$-space dimensions and high acceleration rates.

\section{Funding}

NIH:K99HL111410-01; R01EB008743-01A2. 


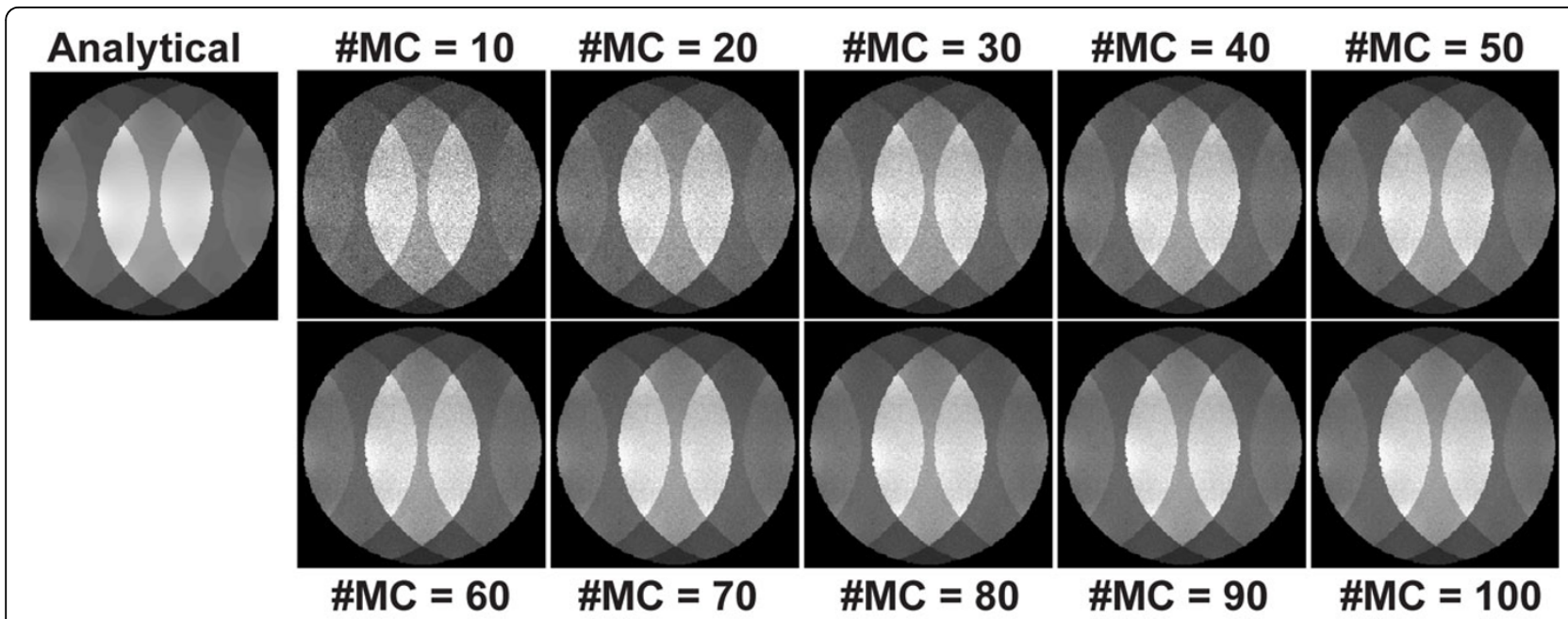

Figure $1 \mathrm{~g}$-factor maps calculated from numerical simulations using point-by-point analytical evaluation, as well as the described Monte-Carlo method for various number of simulations (\#MC). The Monte-Carlo based approach converges after 50 simulations.

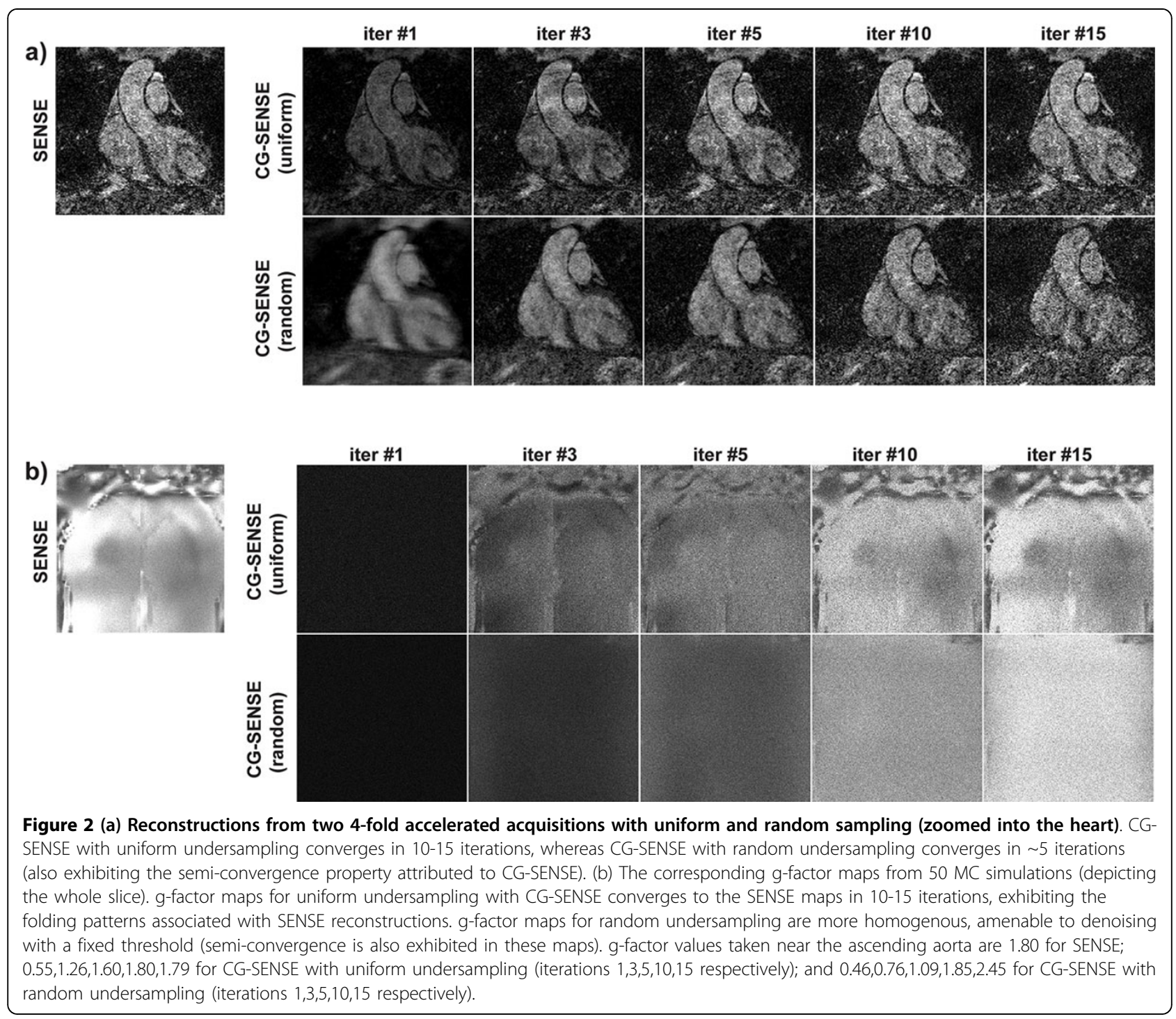




\section{Authors' details}

'Medicine, Beth Israel Deaconess Medical Center, Harvard Medical School,

Boston, Massachusetts, USA. ${ }^{2}$ Radiology, Beth Israel Deaconess Medical

Center, Harvard Medical School, Boston, Massachusetts, USA.

Published: 16 January 2014

\section{References}

1. Pruessmann: MRM 1999.

2. Pruessmann: MRM 2001.

3. Liu: ISMRM 2008.

4. Thunberg: MRI 2007

5. Robson: MRM 2008.

doi:10.1186/1532-429X-16-S1-W28

Cite this article as: Akcakaya et al:: Efficient calculation of g-factors for CG-SENSE in high dimensions: noise amplification in random undersampling. Journal of Cardiovascular Magnetic Resonance 2014 16(Suppl 1):W28.

Submit your next manuscript to BioMed Central and take full advantage of:

- Convenient online submission

- Thorough peer review

- No space constraints or color figure charges

- Immediate publication on acceptance

- Inclusion in PubMed, CAS, Scopus and Google Scholar

- Research which is freely available for redistribution

Submit your manuscript at www.biomedcentral.com/submit
C Biomed Central 